\title{
Cerebellum Tunes the Excitability of the Motor System: Evidence from Peripheral Motor Axons
}

\author{
Hiroyuki Nodera • Mario Manto
}

Published online: 25 June 2014

(C) Springer Science+Business Media New York 2014

\begin{abstract}
Cerebellum is highly connected with the contralateral cerebral cortex. So far, the motor deficits observed in acute focal cerebellar lesions in human have been mainly explained on the basis of a disruption of the cerebello-thalamo-cortical projections. Cerebellar circuits have also numerous anatomical and functional interactions with brainstem nuclei and projects also directly to the spinal cord. Cerebellar lesions alter the excitability of peripheral motor axons as demonstrated by peripheral motor threshold-tracking techniques in cerebellar stroke. The biophysical changes are correlated with the functional scores. Nerve excitability measurements represent an attractive tool to extract the rules underlying the tuning of excitability of the motor pathways by the cerebellum and to discover the contributions of each cerebellar nucleus in this key function, contributing to early plasticity and sensorimotor learning.
\end{abstract}

The tuning of the excitability of the motor system is a key function impacting on early plasticity, sensorimotor learning and recovery from central nervous system (CNS) lesions. Cerebellar circuitry is fundamental for this function. Its cerebellar output emerges from cerebellar nuclei [1]. The output is directed towards the diencephalon, brainstem nuclei and spinal cord [2]. Nuclear projections have a direct impact on the excitability of the motor cortex and premotor areas [3-8]. They also impact directly on the excitability of the spinal cord

\section{H. Nodera}

Department of Neurology, Tokushima University Hospital,

Tokushima, Japan

M. Manto $(\bowtie)$

Unité d'Etude du Mouvement, FNRS-ULB Erasme, 808 Route de Lennik, 1070 Bruxelles, Belgium

e-mail:mmanto@ulb.ac.be circuits [9]. Nuclear projections tune the activity of the corticospinal tract, the reticulospinal tract, the vestibulospinal tract, the tectospinal tract, the rubrospinal tract, the intersticiospinal tract, all belonging to the group of descending tracts reaching the spinal cord [2]. Cerebellar nuclei are involved in reverberating circuits with the brainstem and target nuclei supplying a major input to the inferior olive, this latter projecting itself to the cerebellum via the climbing fibres $[1,2]$.

Overall, cerebellar nuclei exert a facilitatory effect upon the motor cortex. Both the interpositus and dentate nuclei excite the pyramidal tract neurons of the motor cortex [10]. Single pyramidal tract neurons receive convergent inputs from both nuclei, the convergence of the inputs occurring at the level of the ventrolateral nucleus of the thalamus. An excess of inhibitory mechanisms has been demonstrated in the motor cortex of cerebellar patients [5]. The motor threshold (MT, reflecting membrane excitability in pyramidal neurons) of motorevoked potentials (MEPs) is increased contralaterally to a hemicerebellar lesion [6, 7]. The same defect is observed in rodents. In agreement with the prevailing opinion that the major role of the cerebellar cortex is to inhibit cerebellar nuclei which, themselves, facilitate the activity of thalamic nuclei, trains of anodal direct current stimulation (DCS) applied over the motor cortex contralaterally to a hemicerebellar ablation antagonize the decrease in motor cortex excitability, and epidural anodal DCS of the cerebellum (a) decreases the excitability of the motor cortex and (b) enhances the afferent inhibition process associated with peripheral stimuli $[8,9]$.

In human, electrical stimulation of the dentate nucleus and electrocoagulation of the nucleus decrease muscle tone and reduce the amplitudes of $F$ waves (a marker of the excitability of motoneurons, elicited by antidromic activation of peripheral nerves) in the ipsilateral abductor pollicis brevis by $55-60 \%$ [11]. A subsequent dentatotomy on the contralateral side induces a further reduction of $12 \%$ in average. The 
specific effects of cerebellar nuclei stimuli on the excitability of alpha motoneurons are dependent upon the location within the nuclei at which the stimuli are applied. In monkeys, conditioning trains of dentate nucleus stimuli change the post-synaptic potentials evoked in motoneurons by stimulation of group $\mathrm{I} / \mathrm{Ib}$ afferents in given peripheral nerves. Experimental data support the existence of an excitatory cerebellothalamocorticospinal pathway affecting the excitability of motoneurons. Indeed, when a cooling of the motor cortex is applied, the procedure cancels an excitatory component from the intracellular responses evoked in lumbar motoneurons by dentate stimulation [12]. In addition, descending pathways connecting brainstem and cerebellum contribute to the control of muscle tension associated with limb movements. In particular, the dentate nucleus tunes the excitability of spinal neurons via the rapidly conducting reticulospinal pathway [13]. The dentate nucleus thus exerts a significant effect on the excitability of spinal neurons also via pathways which do not include the sensorimotor and premotor cortices. Stimulation of the dentate nucleus changes the amplitude of the inhibitory post-synaptic potential evoked by $\mathrm{Ib}$ afferents in alpha motoneurons. The interpositus nucleus is also an excellent candidate to play a role of modulator of the excitability of the motor system.
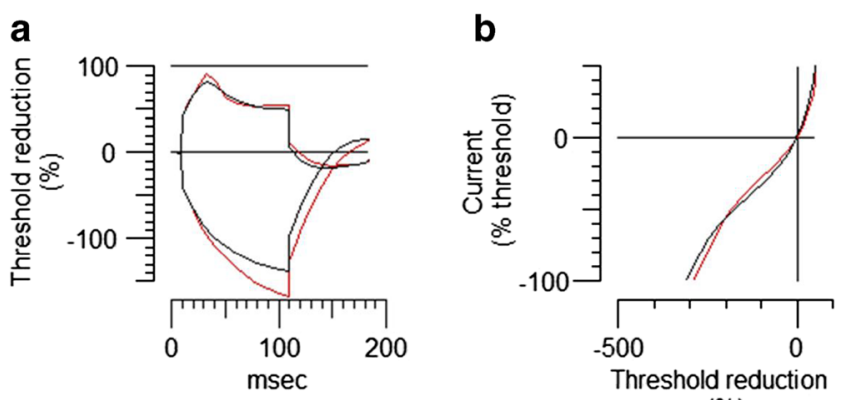

(\%)
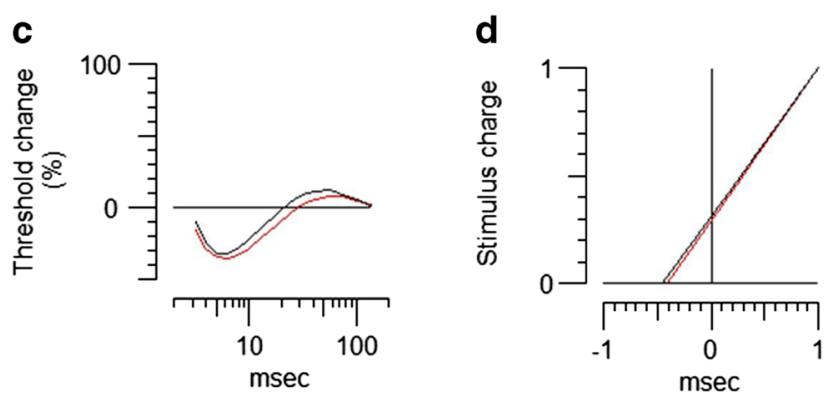

Fig. 1 The waveforms of the threshold tracking techniques and the simulated results obtained by modelling. a The threshold electrotonus (application of long, variable durations of depolarizing and hyperpolarizing conditioning stimuli); b The current threshold response (similar to threshold electrotonus, but with fixed duration of the conditioning stimuli); c The recovery cycle (time course of excitability changes after a single supramaximal stimulation); d Strength-duration time constant (i.e., chronaxie at the intercept of the $x$-axis). Waveform changes shifting from the normal resting membrane potential $(-82.9 \mathrm{mV}$ : black lines $)$ to its hyperpolarized shift ( $-85 \mathrm{mV}$ : red lines) are simulated using modelling
Indeed, this nucleus receives inputs from both sensorimotor cortex and the spinal cord, is involved in somesthetic reflexes, participates in the transformation of sensory signals to motor-oriented commands, and receives climbing fibres mediating highly integrated sensorimotor information derived from spinal modules. Descending fibres originating mainly from the posterior interpositus nucleus (interpositospinal tract) decussate at the mesencephalic level and project caudally to enter the spinal cord, projecting mainly to cervical spinal interneurons, as demonstrated by retrograde transport studies and autoradiography [14]; their terminations are situated on the soma and proximal dendrites of interneurons. Functional blockade of cerebellar interpositus nucleus with tetrodotoxin (TTX) reduces the slope of the $H$ reflex recruitment curve without affecting the $H \max / M \max$ ratios, and depresses both $F$ wave persistence and mean $F /$ mean $M$ ratios [15]. While the interpositospinal tract terminates on interneurons, the fastigiospinal neurons excite motoneurons monosynaptically [14]. The importance of the nucleo-spinal projections in the understanding of cerebellar signs might have likely been underestimated so far.

Peripheral motor threshold tracking techniques are similar to nerve conduction studies, with the exception of using various strengths and durations of conditioning stimuli to assess the reactions of the motor axons that reflect the overall excitability, the resting membrane potential and functions of various ion channels (Fig. 1). With the aid of these techniques, cerebellar lesions impair the excitability of peripheral motor axons as demonstrated in a longitudinal study of first-time unilateral cerebellar stroke [16]. The patients investigated did not exhibit any clinical or radiological evidence of motor tract involvement. The changes developed at a peripheral level early after stroke onset. Abnormal stimulus thresholds, rheobase and threshold electrotonus curves were observed, all of which suggested hyperpolarized shift of the resting membrane potential of the peripheral motor axon. Very interestingly, the biophysical changes were correlated with the functional deficits observed in patients and recover as the patients improve clinically. Transynaptic plasticity consecutive to the cerebellar lesion is suggested.

Nerve excitability measurements represent an attractive tool to quantify the tuning of excitability of the motor pathways by the cerebellum. This technique will contribute to the discovery of the contributions of cerebellar nuclei in motor control. Whether this tool will emerge as a biomarker of cerebellar function remains to be established; combinations of assessments with non-invasive techniques of stimulation of the CNS are anticipated.

Acknowledgments MM is supported by the FNRS-Belgium.

Conflicts of Interest The authors report no conflict of interest. 


\section{References}

1. Ruigrok TJ. Ins and outs of the cerebellar modules. Cerebellum. 2011;10:464-74.

2. Ruigrok TJ. Cerebellar influences on descending spinal motor systems. In: Manto M, Gruol DL, Schmahmann JD, Koibuchi N, Rossi F, editors. Handbook of cerebellum and cerebellar disorders. Doordrecht: Springer; 2013. p. 497-528.

3. Kuypers HGJM. Anatomy of the descending pathways. In: Brookhart JM, Mountcastle VB, Brooks VB, Geiger SR, editors. Handbook of physiology, Motor control, part 1, vol. II. Bethesda: The American Physiological Society; 1981. p. 597-666.

4. Tsukahara N, Bando T, Murakami F, Oda Y. Properties of cerebello-precerebellar reverberating circuits. Brain Res. 1983;274:249-59.

5. Tamburin S, Fiaschi A, Andreoli A, Marani S, Manganotti P, Zanette G. Stimulus-response properties of motor system in patients with cerebellar ataxia. Clin Neurophysiol. 2004;115(2):348-55.

6. Di Lazzaro V, Restuccia D, Nardone R, Leggio MG, Oliviero A, Profice $\mathrm{P}$, et al. Motor cortex changes in a patient with hemicerebellectomy. Electroencephalogr Clin Neurophysiol. 1995;97(5):259-63.

7. Ziemann U, Lönnecker S, Steinhoff BJ, Paulus W. Effects of antiepileptic drugs on motor cortex excitability in humans: a transcranial magnetic stimulation study. Ann Neurol. 1996;40(3): $367-78$.
8. Oulad Ben Taib N, Manto M. Trains of transcranial direct current stimulation antagonize motor cortex hypoexcitability induced by acute hemicerebellectomy. J Neurosurg. 2009;111:796-806.

9. Oulad Ben Taib N, Manto M. Trains of epidural DC stimulation of the cerebellum tune corticomotor excitability. Neural Plast 2013;613197. doi:10.1155/2013/613197

10. Shinoda Y, Yamazaki M, Futami T. Convergent inputs from the dentate and the interpositus nuclei to pyramidal tract neurons in the motor cortex. Neurosci Lett. 1982;34(2):111-5.

11. Fox JE, Hitchcock ER. Changes in F wave size during dentatotomy. $\mathrm{J}$ Neurol Neurosurg Psychiatry. 1982;45(12):1165-7.

12. Bantli H, Bloedel J. The action of the dentate nucleus on the excitability of spinal motoneurons via pathways which do not involve the primary sensorimotor cortex. Brain Res. 1975;88:86-90.

13. Bantli H, Bloedel JR. Monosynaptic activation of a direct reticulospinal pathway by the dentate nucleus. Pflugers Arch. 1975;357(34):237-42.

14. Asanuma $\mathrm{C}$, Thach $\mathrm{W}$, Jones EG. Nucleus interpositus projection to spinal interneurons in monkey. Brain Res. 1980;191:245-8.

15. Oulad Ben Taib N, Laute MA, Pandolfo M, Manto MU. Interaction between repetitive stimulation of the sciatic nerve and functional ablation of cerebellar nucleus interpositus in the rat. Cerebellum. 2004;3:21-6.

16. Huynh W, Lin CSY, Krishnan AV, Vucic S, Kiernan MC. Transynaptic changes evident in peripheral axonal function after acute cerebellar infarct. Cerebellum, this issue. 